\title{
Challenge for spontaneous $C P$ violation in Type IIB orientifolds with fluxes
}

\author{
Tatsuo Kobayashi ${ }^{1, *}$ and Hajime Otsuka ${ }^{2, \uparrow}$ \\ ${ }^{1}$ Department of Physics, Hokkaido University, Sapporo 060-0810, Japan \\ ${ }^{2}$ KEK Theory Center, Institute of Particle and Nuclear Studies, \\ KEK, 1-1 Oho, Tsukuba, Ibaraki 305-0801, Japan
}

(Received 28 May 2020; accepted 18 June 2020; published 7 July 2020)

\begin{abstract}
We systematically investigate the possibilities of realizing the spontaneous $C P$ violation in Type IIB flux compactifications on toroidal orientifolds. Our detailed analysis for a generic choice of three-form fluxes leads to the presence of flat directions, where $C P$-breaking and -conserving vacua are degenerate, indicating that flux compactifications are not sufficient to realize the spontaneous $C P$ violation. Furthermore, the four-dimensional $C P$ can be embedded into the duality symmetries, namely modular symmetries for a particular choice of fluxes.
\end{abstract}

DOI: 10.1103/PhysRevD.102.026004

\section{INTRODUCTION}

Understanding the origin of $C P$ violation in the Standard Model (SM) is of particular interest, not only to explain the structure of Yukawa couplings but also to search for new physics beyond the SM through experiments on the neutron electric dipole moment [1], $K$ meson decays (probed by the KOTO experiment at the J-PARC and the NA62 experiments at CERN), and observations of the excess of baryons over antibaryons in the Universe. It is interesting to ask the origin of $C P$ violation in string theory.

As pointed out in Refs. [2,3], $C P$ is regarded as a discrete gauge symmetry in a class of $E_{8} \times E_{8}$ and $S O(32)$ heterotic string theories. Calabi-Yau (CY) compactifications lead to the spontaneous breaking of $C P$ by the dynamics of massless scalar fields, called moduli fields [4]. Here, the four-dimensional (4D) $C P$ is embedded into the $10 \mathrm{D}$ proper Lorentz symmetry, indicating that the orientations of 4D spacetime and 6D CY threefolds are reversed simultaneously. In the heterotic string context, axionic components of an axiodilaton and Kähler moduli have an odd parity under $C P$ transformation [4] and the 4D $C P$ can be spontaneously broken by their vacuum expectation values (vevs). However, it is difficult to achieve the stabilization of the moduli fields on CY threefolds as well

\footnotetext{
kobayashi@particle.sci.hokudai.ac.jp

†hotsuka@post.kek.jp
}

Published by the American Physical Society under the terms of the Creative Commons Attribution 4.0 International license. Further distribution of this work must maintain attribution to the author(s) and the published article's title, journal citation, and DOI. Funded by SCOAP ${ }^{3}$. as toroidal orbifolds in a controlled way, due to sizable backreactions from their stabilization. ${ }^{1}$

The purpose of this paper is to systematically investigate the possibilities of realizing the spontaneous $C P$ violation in Type IIB string theory on toroidal orientifolds, where the moduli stabilization as well as the calculation of Yukawa couplings is performed in a controlled way. Similar to the heterotic string theory, $C P$-odd axionic fields break the $4 \mathrm{D}$ $C P$ spontaneously, and their vevs induce the nonvanishing Cabbibo-Kobayashi-Maskawa (CKM) phase. Since it is possible to stabilize these axionic fields by three-form fluxes in the low-energy effective action of Type IIB string theory, we search for $C P$-breaking minima in Type IIB flux compactifications on several toroidal orientifolds. Starting from a $C P$-invariant potential with a generic choice of three-form fluxes, we find that flat directions exist and $C P$ breaking and -conserving vacua are degenerate. To realize the spontaneous $C P$ violation, a resolution of this degeneracy by other sources is required. Furthermore, 4D $C P$ can be embedded into the duality symmetries, namely modular symmetries for a particular choice of fluxes. The approach to unify $C P$ and modular symmetries is recently developed in Refs. [8-11], and the relationship between the strong $C P$ and CKM phases is also pointed out in Ref. [12]. Indeed, axions associated with the complex structure moduli of tori play a crucial role in such a generalized $C P$ context [13-16].

This paper is organized as follows. In Sec. II, we briefly review the origin of $4 \mathrm{D} C P$ in the effective action of Type II string theory with intersecting/magnetized $D$-branes. In Sec. III, we discuss the $C P$-invariant low-energy effective

\footnotetext{
${ }^{1}$ It might be possible to break $C P$ through vevs of charged scalar fields under the anomalous $\mathrm{U}(1)$ symmetry [5]. For the $C P$ violation in the orbifold context, see Refs. [6,7].
} 
action of Type IIB string theory on toroidal orientifolds. Three-form fluxes cause both the stabilization of axionic fields and spontaneous breaking of $C P$. It turns out that massless fields generically appear in the flux vacua on several toroidal orientifolds. Finally, we conclude in Sec. IV.

\section{ORIGIN OF CP IN INTERSECTING/ MAGNETIZED $D$-BRANE MODELS}

In this section, we review the low-energy effective action of magnetized $D$-branes in Type IIB string theory on toroidal orientifolds with a special emphasis on 4D $C P$. It is applicable to the Type IIA intersecting $D$-brane system by T-dualizing Type IIB magnetized $D$-brane models.

Let us consider magnetized $D 7$-branes wrapping $\left(T^{2}\right)_{i} \times$ $\left(T^{2}\right)_{j}$ with $i \neq j$. When $U(1)$ gauge boson on $D 7$-branes takes a nontrivial vev on the torus, the internal space carries background gauge flux $F^{i}$ on each torus $\left(T^{2}\right)_{i}$,

$$
\frac{m^{i}}{l_{s}^{2}} \int_{\left(T^{2}\right)_{i}} F^{i}=n^{i}
$$

where $m^{i}$ and $n^{i}$ correspond to the wrapping number of magnetized $D 7$-branes and the flux quanta, respectively. $l_{s}=2 \pi \sqrt{\alpha^{\prime}}$ denotes the string length. Such gauge fluxes are relevant to determine $4 \mathrm{D} C P$ as discussed later.

The 4D $\theta$ term in the effective action of $D 7$-branes is determined by the following gauge kinetic function [17-19]:

$$
f_{\mathrm{D} 7}=\left|m^{i} m^{j}\right|\left(T^{k}-\frac{n^{i} n^{j}}{m^{i} m^{j}} S\right)
$$

with $i \neq j \neq k$. Here, we denote the Kähler moduli $T^{i}$ and the axiodilaton $S$, respectively. Remarkably, the $C P$-odd axionic components of these moduli fields (RamondRamond fields) $\operatorname{Re}\left(T^{i}\right)=\int_{\left(T^{2}\right)_{j} \times\left(T^{2}\right)_{k}} C_{4}$ and $\operatorname{Re}(S)=C_{0}$ induce the 4D $\theta$ term.

The CKM phase in Yukawa couplings of chiral zero modes on magnetized $D 7$-branes is also determined by one of the closed string moduli, namely the complex structure moduli $\tau_{i}$ associated with tori $\left(T^{2}\right)_{i}$. Indeed, the presence of magnetic fluxes induces the multiple number of chiral zero modes with nontrivial moduli-dependent Yukawa couplings. From the calculation of the Yukawa couplings of chiral zero modes in the conformal field theory as well as the field theoretical approach, it is known that holomorphic Yukawa couplings on each two torus $\left(T^{2}\right)_{j}$ inside the fourcycle wrapped by magnetized $D 7$-branes are given by the Jacobi theta function [20-23],

$$
\vartheta\left[\begin{array}{l}
c \\
0
\end{array}\right]\left(0, \tau_{j}\right) \equiv \sum_{l \in \mathbb{Z}} e^{\pi i(c+l)^{2} \tau_{j}}
$$

For illustrative purposes, let us suppose that the $U(N)$ gauge symmetry realized in $N$ stacks of $D 7$-branes is broken by magnetic fluxes to $U\left(N_{a}\right) \times U\left(N_{b}\right) \times U\left(N_{c}\right)$ with $N=N_{a}+N_{b}+N_{c}$. Then, bifundamental zero modes $\left(N_{\alpha}, \bar{N}_{\beta}\right), \alpha, \beta=a, b, c$ have $I_{\alpha \beta}^{i}=n_{\alpha}^{i} / m_{\alpha}^{i}-n_{\beta}^{i} / m_{\beta}^{i}$ number of zero modes on each torus $\left(T^{2}\right)_{i}$. Holomorphic Yukawa couplings of such zero modes are provided by

$$
Y_{p q s}=\vartheta\left[\begin{array}{c}
-\frac{1}{I_{a b}^{i}}\left(\frac{q}{I_{c a}^{i}}+\frac{s}{I_{b c}^{i}}\right) \\
0
\end{array}\right]\left(0, \tau_{i}\left|I_{a b}^{i} I_{b c}^{i} I_{c a}^{i}\right|\right),
$$

up to the normalization factor. Here, $q=0,1, \ldots,\left|I_{c a}^{i}\right|-1$, and $s=0,1, \ldots,\left|I_{b c}^{i}\right|-1$ denote flavor indices, and we employ $p=s-q \bmod I_{a b}$ [23].

In this way, axionic components of the complex structure moduli determine the magnitude of CKM phase, namely the Jarlskog determinant. (For explicit examples, see, e.g., Ref. [24].) The moduli-dependent Yukawa couplings as well as the gauge kinetic function in Type IIA intersecting $D 6$-branes have the analogous form by T-dualizing the Type IIB magnetized $D 7$-brane models [20]. For Type IIB string theory with $D 5 / D 9$-branes, the gauge kinetic function and Yukawa couplings are also dependent on the Kähler moduli and complex structure moduli, respectively. It is remarkable that the complex structure moduli control not only the 4D $C P$ but also the flavor symmetry in Type IIB string theory. ${ }^{2}$

\section{FOUR-DIMENSIONAL $C P$ IN FLUX COMPACTIFICATIONS}

So far, we have discussed the origin of 4D $C P$ phases in the effective action of magnetized $D$-branes. This section is devoted to exploring the spontaneous $C P$ violation on the basis of the effective action of moduli fields. We draw general conclusions about the spontaneous $C P$ violation in Type IIB toroidal orientifolds with fluxes.

\section{A. $C P$-invariant moduli potential}

We begin with the simplest $T^{6} / \mathbb{Z}_{2}$ orientifold, following the convention of Ref. [27]. It is straightforward to extend the setup to $T^{6} /\left(\mathbb{Z}_{2} \times \mathbb{Z}_{2}^{\prime}\right)$ and other orbifolds, as discussed later.

As argued in Refs. [2,3], the 4D $C P$ is regarded as the higher-dimensional proper Lorentz symmetry, in particular, the 10D proper Lorentz symmetry in string theory. When the extra $6 \mathrm{D}$ space consists of the factorizable $T^{6}$ subject to

\footnotetext{
${ }^{2}$ For the relationship between the flavor symmetry and the modular symmetry of $\tau$ in Type IIB string theory, see, e.g., Refs. [25,26].
} 
$\mathbb{Z}_{2}$ identification, namely $\Pi_{i=1}^{3}\left(T^{2}\right)_{i} / \mathbb{Z}_{2}$ with coordinates $z_{i}$, the 10D proper Lorentz symmetry is defined by the 4D $C P$ and $6 \mathrm{D}$ transformations $z_{i} \rightarrow z_{i}^{*}$ or $z_{i} \rightarrow-z_{i}^{*}$, leading to the negative determinant in the transformation of $6 \mathrm{D}$ space. In this paper, we restrict ourselves to $z_{i} \rightarrow-z_{i}^{*}$ transformations, allowing us to consider $\operatorname{Im} \tau_{i}>0$ in the complex structure moduli spaces. According to $z_{i} \rightarrow-z_{i}^{*}, \tau_{i}$ transform as

$$
\tau_{i} \rightarrow-\bar{\tau}_{i}
$$

and at the same time, the axiodilaton $S=C_{0}+i e^{-\phi}$ also transforms

$$
S \rightarrow-\bar{S}
$$

Note that the real part of the axiodilaton is the RamondRamond axion.

Let us discuss $C P$ invariance of the moduli effective action on the basis of $\Pi_{i=1}^{3}\left(T^{2}\right)_{i} / \mathbb{Z}_{2}$ orientifold. Under the above transformations, the moduli Kähler potential,

$$
\begin{aligned}
K= & -\ln (-i(S-\bar{S}))-2 \ln \mathcal{V} \\
& -\ln \left(i\left(\tau_{1}-\bar{\tau}_{1}\right)\left(\tau_{2}-\bar{\tau}_{2}\right)\left(\tau_{3}-\bar{\tau}_{3}\right)\right)
\end{aligned}
$$

is invariant. Here, we denote $\mathcal{V}$ as the volume of $T^{6}$ and employ the reduced Planck mass unit $M_{\mathrm{Pl}}=1$. On the other hand, the superpotential is induced by an existence of a background three-form flux $G_{3}$ [28],

$$
W=\frac{1}{l_{s}^{2}} \int \Omega \wedge G_{3},
$$

with $l_{s}=2 \pi \sqrt{\alpha^{\prime}}$. $^{3}$ Now the holomorphic three-form $\Omega$,

$$
\Omega=d z_{1} \wedge d z_{2} \wedge d z_{3}
$$

transforms under the $C P$ transformation as

$$
\Omega \rightarrow-\bar{\Omega}
$$

The three-form flux $G_{3}$ is defined by a linear combination of Ramond-Ramond (RR) $F_{3}$ and Neveu-Schwarz (NS) three-forms $\mathrm{H}_{3}$, namely $\mathrm{G}_{3}=\mathrm{F}_{3}-\mathrm{SH}_{3}$; both of which are expanded on the basis of $H^{3}\left(T^{6}, \mathbb{Z}\right)$,

\footnotetext{
${ }^{3}$ The normalization factor to match with the dimensional reduction of Type IIB supergravity action is irrelevant to the following discussion, and therefore, we omit such a factor for simplicity.
}

$$
\begin{aligned}
& \frac{1}{l_{s}^{2}} F_{3}=a^{0} \alpha_{0}+a^{i} \alpha_{i}+b_{i} \beta^{i}+b_{0} \beta^{0}, \\
& \frac{1}{l_{s}^{2}} H_{3}=c^{0} \alpha_{0}+c^{i} \alpha_{i}+d_{i} \beta^{i}+d_{0} \beta^{0},
\end{aligned}
$$

with

$$
\begin{array}{ll}
\alpha_{0}=d x^{1} \wedge d x^{2} \wedge d x^{3}, & \alpha_{1}=d y^{1} \wedge d x^{2} \wedge d x^{3}, \\
\alpha_{2}=d y^{2} \wedge d x^{3} \wedge d x^{1}, & \alpha_{3}=d y^{3} \wedge d x^{1} \wedge d x^{2}, \\
\beta^{0}=d y^{1} \wedge d y^{2} \wedge d y^{3}, & \beta^{1}=-d x^{1} \wedge d y^{2} \wedge d y^{3}, \\
\beta^{2}=-d x^{2} \wedge d y^{3} \wedge d y^{1}, & \beta^{3}=-d x^{3} \wedge d y^{1} \wedge d y^{2},
\end{array}
$$

satisfying $\int_{T^{6}} \alpha_{I} \wedge \beta^{J}=\delta_{I}^{J}$. Note that flux quanta $\left\{a^{0,1,2,3}, b_{0,1,2,3}, c^{0,1,2,3}, d_{0,1,2,3}\right\}$ are even and/or odd integers depending on the existence of exotic $\mathrm{O}^{\prime}$-planes $[29,30]$, and they are constrained by the tadpole cancellation condition,

$$
\begin{aligned}
n_{\mathrm{flux}} & =\frac{1}{l_{s}^{2}} \int H_{3} \wedge F_{3} \\
& =c^{0} b_{0}-d_{0} a^{0}+\sum_{i}\left(c^{i} b_{i}-d_{i} a^{i}\right) \\
& =32-2 n_{\mathrm{D} 3}-n_{\mathrm{O} 3^{\prime}} \leq 32,
\end{aligned}
$$

where $n_{\mathrm{D} 3}$ and $n_{\mathrm{O}^{\prime}}$ are the number of $D 3$-branes and exotic O3'-planes, respectively. Here, anti-D3-brane contributions are not taken into account to preserve the supersymmetry in our system.

Since the coordinate transformations $z_{i} \rightarrow-z_{i}^{*}$ correspond to $x_{i} \rightarrow-x_{i}$ and $y_{i} \rightarrow y_{i}$, these three-form bases also transform as

$$
\begin{gathered}
\alpha_{0} \rightarrow-\alpha_{0}, \quad \beta^{0} \rightarrow \beta^{0}, \\
\alpha_{i} \rightarrow \alpha_{i}, \quad \beta^{i} \rightarrow-\beta^{i}, \quad(i=1,2,3) .
\end{gathered}
$$

To be invariant under the $C P$ transformation, the fluxinduced superpotential should be transformed as

$$
W \rightarrow e^{i \gamma} \bar{W},
$$

with $\gamma=0$ or $\pi(\bmod 2 \pi)$ because $F_{3}$ and $H_{3}$ are integer quantized and have no imaginary part. Recall that the only complex quantity is $S$ in $G_{3}$, with $\gamma \neq 0$, and $\pi(\bmod 2 \pi)$ is contradicted with the real three-forms $F_{3}$ and $H_{3}$. Hence, the three-form fluxes obey

$$
G_{3} \rightarrow-e^{i \gamma} \bar{G}_{3} .
$$

It restricts us to the following two patterns of RR and NSNS three-forms: 
(i) $\gamma=0(\bmod 2 \pi)$

$$
\begin{aligned}
& \frac{1}{l_{s}^{2}} F_{3}=a^{0} \alpha_{0}+b_{i} \beta^{i}, \\
& \frac{1}{l_{s}^{2}} H_{3}=c^{i} \alpha_{i}+d_{0} \beta^{0},
\end{aligned}
$$

leading to $G_{3} \rightarrow-F_{3}+\bar{S} H_{3}=-\bar{G}_{3}$.

(ii) $\gamma=\pi(\bmod 2 \pi)$

$$
\begin{aligned}
& \frac{1}{l_{s}^{2}} F_{3}=a^{i} \alpha_{i}+b_{0} \beta^{0}, \\
& \frac{1}{l_{s}^{2}} H_{3}=c^{0} \alpha_{0}+d_{i} \beta^{i},
\end{aligned}
$$

leading to $G_{3} \rightarrow F_{3}-\bar{S} H_{3}=\bar{G}_{3}$. Other choices to obtain the nonvanishing $\mathrm{CP}$-invariant superpotential are impossible because of the real three-forms $F_{3}$ and $H_{3}$.

Then, it results in the two classes of the superpotential:

(i) $\gamma=0(\bmod 2 \pi)$

$$
\begin{aligned}
W= & a^{0} \tau_{1} \tau_{2} \tau_{3}+c^{1} S \tau_{2} \tau_{3}+c^{2} S \tau_{1} \tau_{3} \\
& +c^{3} S \tau_{1} \tau_{2}-\sum_{i=1}^{3} b_{i} \tau_{i}+d_{0} S
\end{aligned}
$$

(ii) $\gamma=\pi(\bmod 2 \pi)$

$$
\begin{aligned}
W= & -c^{0} S \tau_{1} \tau_{2} \tau_{3}-a^{1} \tau_{2} \tau_{3}-a^{2} \tau_{1} \tau_{3} \\
& -a^{3} \tau_{1} \tau_{2}+\sum_{i=1}^{3} d_{i} S \tau_{i}-b_{0} .
\end{aligned}
$$

Note that the above statement also holds for $\Pi_{i=1}^{3}\left(T^{2}\right)_{i} /\left(\mathbb{Z}_{2} \times \mathbb{Z}_{2}^{\prime}\right)$ orientifolds with or without $D$ branes, taking into account the expansion of $F_{3}, H_{3}$ on the basis of $H_{*}^{3}\left(T^{6}, 4 \mathbb{Z}\right)$ for the case without discrete torsion, and $H_{*}^{3}\left(T^{6}, 8 \mathbb{Z}\right)$ for the case with a discrete torsion. Here, the $\Omega_{3}$ and $G_{3}$ are expanded with respect to a basis of $H_{-}^{3}\left(T^{6}\right)$ for the orientifold with $O 3 / O 7$-planes and $H_{+}^{3}\left(T^{6}\right)$ for the orientifold with $O 5 / O 9$-planes with $H_{3}=0$, where the number of untwisted moduli is counted by $h_{-}^{2,1}$ and $h_{+}^{2,1}$, respectively. (For the detailed discussions of toroidal orientifolds, see, e.g., Ref. [31].) Other toroidal orbifolds are discussed in Sec. III D.

\section{B. $T^{6} / \mathbb{Z}_{2}$ and $T^{6} /\left(\mathbb{Z}_{2} \times \mathbb{Z}_{2}^{\prime}\right)$ with single complex structure modulus}

We first analyze the overall complex structure modulus, namely $\tau \equiv \tau_{1}=\tau_{2}=\tau_{3}$, and check whether the $4 \mathrm{D} C P$ is spontaneously broken or not. For the single complex structure modulus, the superpotential is simplified as

$$
W=\left\{\begin{array}{cc}
a^{0} \tau^{3}+3 c S \tau^{2}-3 b \tau+d_{0} S, & (\gamma=0) \\
-c^{0} S \tau^{3}-3 a \tau^{2}+3 d S \tau-b_{0}, & (\gamma=\pi)
\end{array},\right.
$$

where we define

$a \equiv a^{1}=a^{2}=a^{3}, \quad b \equiv b_{1}=b_{2}=b_{3}$,

$c \equiv c^{1}=c^{2}=c^{3}, \quad d \equiv d_{1}=d_{2}=d_{3}$.

On the other hand, the Kähler potential is given by

$$
K=-\ln (-i(S-\bar{S}))-2 \ln \mathcal{V}-3 \ln (-i(\tau-\bar{\tau})) .
$$

As a consequence of the no-scale structure for the volume moduli, the moduli fields except for the volume moduli can be stabilized at the supersymmetric minimum $D_{\tau} W=$ $D_{S} W=0$ with $D_{I} W=\partial_{I} W+\left(\partial_{I} K\right) W$ [32],

(i) $\gamma=0(\bmod 2 \pi)$

$$
\begin{gathered}
\operatorname{Re}(\tau)=\operatorname{Re}(S)=0, \quad \operatorname{Im}(\tau)=\left(-\frac{b d_{0}}{a^{0} c}\right)^{1 / 4}, \\
\operatorname{Im}(S)=\left(-\frac{a^{0}(b)^{3}}{(c)^{3} d_{0}}\right)^{1 / 4},
\end{gathered}
$$

(ii) $\gamma=\pi(\bmod 2 \pi)$

$$
\begin{gathered}
\operatorname{Re}(\tau)=\operatorname{Re}(S)=0, \quad \operatorname{Im}(\tau)=\left(-\frac{b_{0} d}{a c^{0}}\right)^{1 / 4}, \\
\operatorname{Im}(S)=\left(-\frac{(a)^{3} b_{0}}{c^{0}(d)^{3}}\right)^{1 / 4} .
\end{gathered}
$$

As a result, the 4D $C P$ is not spontaneously broken at the supersymmetric minimum for this simplest case. Hence, we move on to the case treating three independent complex structure moduli and search for the supersymmetric $C P$ breaking minimum in the next section.

\section{C. $T^{6} / \mathbb{Z}_{2}$ and $T^{6} /\left(\mathbb{Z}_{2} \times \mathbb{Z}_{2}^{\prime}\right)$ with three complex structure moduli}

We next explore the existence of a $C P$-breaking minimum for the case with three complex structure moduli. We analytically discuss the spontaneous $C P$ violation for $\gamma=0$ in Sec. III C 1 and $\gamma=\pi$ in Sec. III C 2, respectively.

\section{Odd polynomials $(\gamma=0)$}

In this section, we focus on the superpotential (19) consisting of odd polynomials with respect to the moduli fields. The supersymmetric conditions $D_{I} W=0$ are still complicated equations with respect to the moduli fields, even for the $C P$ invariant superpotential (19). To analytically solve the supersymmetric conditions, we simplify the effective action by redefining the moduli fields, 


$$
\tau_{1}=\frac{c^{1}}{a^{0}} x_{s} \tau_{1}^{\prime}, \quad \tau_{2}=\frac{c^{2}}{a^{0}} x_{s} \tau_{2}^{\prime}, \quad \tau_{3}=\frac{c^{3}}{a^{0}} x_{s} \tau_{3}^{\prime}, \quad S=x_{s} S^{\prime}
$$

According to the redefinition of the moduli fields, the Kähler potential and the superpotential are redefined as

$$
\begin{aligned}
K & =-\ln (-i(S-\bar{S}))-2 \ln \mathcal{V}-\ln \left(i\left(\tau_{1}-\bar{\tau}_{1}\right)\left(\tau_{2}-\bar{\tau}_{2}\right)\left(\tau_{3}-\bar{\tau}_{3}\right)\right) \\
& =-\ln \left(-i\left(S^{\prime}-\bar{S}^{\prime}\right)\right)-2 \ln \mathcal{V}-\ln \left(i\left(\tau_{1}^{\prime}-\bar{\tau}_{1}^{\prime}\right)\left(\tau_{2}^{\prime}-\bar{\tau}_{2}^{\prime}\right)\left(\tau_{3}^{\prime}-\bar{\tau}_{3}^{\prime}\right)\right)-\ln \left(x_{s}^{4} c^{1} c^{2} c^{3} /\left(a^{0}\right)^{3}\right) \\
W & =a^{0} \tau_{1} \tau_{2} \tau_{3}+c^{1} S \tau_{2} \tau_{3}+c^{2} S \tau_{1} \tau_{3}+c^{3} S \tau_{1} \tau_{2}-\sum_{i=1}^{3} b_{i} \tau_{i}+d_{0} S \\
& =\left\{\tau_{1}^{\prime} \tau_{2}^{\prime} \tau_{3}^{\prime}+S^{\prime} \tau_{2}^{\prime} \tau_{3}^{\prime}+S^{\prime} \tau_{1}^{\prime} \tau_{3}^{\prime}+S^{\prime} \tau_{1}^{\prime} \tau_{2}^{\prime}\right\}-\sum_{i=1}^{3} b_{i}^{\prime} \tau_{i}^{\prime}+d_{0}^{\prime} S^{\prime}
\end{aligned}
$$

where we take

$$
x_{s}=\left(\frac{\left(a^{0}\right)^{2}}{c^{1} c^{2} c^{3}}\right)^{1 / 3}, \quad b_{i}^{\prime}=b_{i} \frac{c^{i}}{a^{0}} x_{s}, \quad d_{0}^{\prime}=d_{0} x_{s} .
$$

In the following analysis, we omit prime symbols of fields unless specified otherwise. Then, we analyze the following Kähler potential and superpotential:

$$
\begin{aligned}
& K=-\ln (-i(S-\bar{S}))-2 \ln \mathcal{V}-\ln \left(i\left(\tau_{1}-\bar{\tau}_{1}\right)\left(\tau_{2}-\bar{\tau}_{2}\right)\left(\tau_{3}-\bar{\tau}_{3}\right)\right), \\
& W=\tau_{1} \tau_{2} \tau_{3}+S \tau_{2} \tau_{3}+S \tau_{1} \tau_{3}+S \tau_{1} \tau_{2}-\sum_{i=1}^{3} b_{i} \tau_{i}+d_{0} S
\end{aligned}
$$

which is a simplified version of the superpotential (19) with $a^{0}=c^{1}=c^{2}=c^{3}=1$.

To analytically solve the supersymmetric conditions $D_{I} W=0$, we analyze the following equations equivalent to solve $D_{I} W=0$ :

$$
\begin{gathered}
\operatorname{Im}\left(\tau_{1}\right) \operatorname{Re}\left(D_{\tau_{1}} W\right) \pm \operatorname{Im}\left(\tau_{2}\right) \operatorname{Re}\left(D_{\tau_{2}} W\right) \pm \operatorname{Im}\left(\tau_{3}\right) \operatorname{Re}\left(D_{\tau_{3}} W\right)-\operatorname{Im}(S) \operatorname{Re}\left(D_{S} W\right)=0, \\
\operatorname{Im}\left(\tau_{1}\right) \operatorname{Re}\left(D_{\tau_{1}} W\right) \mp \operatorname{Im}\left(\tau_{2}\right) \operatorname{Re}\left(D_{\tau_{2}} W\right) \pm \operatorname{Im}\left(\tau_{3}\right) \operatorname{Re}\left(D_{\tau_{3}} W\right)+\operatorname{Im}(S) \operatorname{Re}\left(D_{S} W\right)=0, \\
\operatorname{Im}\left(\tau_{1}\right) \operatorname{Im}\left(D_{\tau_{1}} W\right) \pm \operatorname{Im}\left(\tau_{2}\right) \operatorname{Im}\left(D_{\tau_{2}} W\right)+\operatorname{Im}\left(\tau_{3}\right) \operatorname{Im}\left(D_{\tau_{3}} W\right) \pm \operatorname{Im}(S) \operatorname{Im}\left(D_{S} W\right)=0, \\
\operatorname{Im}\left(\tau_{1}\right) \operatorname{Im}\left(D_{\tau_{1}} W\right) \mp \operatorname{Im}\left(\tau_{2}\right) \operatorname{Im}\left(D_{\tau_{2}} W\right)-\operatorname{Im}\left(\tau_{3}\right) \operatorname{Im}\left(D_{\tau_{3}} W\right) \pm \operatorname{Im}(S) \operatorname{Im}\left(D_{S} W\right)=0,
\end{gathered}
$$

where the \pm signs are correlated. There is a $C P$-conserving solution,

$$
\begin{aligned}
& \operatorname{Re}\left(\tau_{1}\right)=\operatorname{Re}\left(\tau_{2}\right)=\operatorname{Re}\left(\tau_{3}\right)=\operatorname{Re}(S)=0, \quad \operatorname{Im} \tau_{1}=\left(-\frac{b_{2} b_{3} d_{0}}{b_{1}}\right)^{1 / 4}, \\
& \operatorname{Im}\left(\tau_{2}\right)=\left(-\frac{b_{1} b_{3} d_{0}}{b_{2}}\right)^{1 / 4}, \quad \operatorname{Im}\left(\tau_{3}\right)=\left(-\frac{b_{1} b_{2} d_{0}}{b_{3}}\right)^{1 / 4}, \quad \operatorname{Im}(S)=\left(-\frac{b_{1} b_{2} b_{3}}{d_{0}}\right)^{1 / 4},
\end{aligned}
$$

at which all the moduli masses squared are positive. In addition, we find that there exist five classes of solutions enumerated as follows: 
(i) Solution 1

$$
\begin{aligned}
& \frac{\operatorname{Re}\left(\tau_{2}\right)}{\operatorname{Re}\left(\tau_{1}\right)}=\frac{\operatorname{Im}\left(\tau_{2}\right)}{\operatorname{Im}\left(\tau_{1}\right)}=\sqrt{\frac{b_{1}}{b_{2}}}, \quad \frac{\operatorname{Re}\left(\tau_{3}\right)}{\operatorname{Re}\left(\tau_{1}\right)}=-\frac{\operatorname{Im}\left(\tau_{3}\right)}{\operatorname{Im}\left(\tau_{1}\right)}=-\sqrt{\frac{b_{1}}{b_{3}}}, \\
& \frac{\operatorname{Re}(S)}{\operatorname{Re}\left(\tau_{1}\right)}=\frac{\operatorname{Im}(S)}{\operatorname{Im}\left(\tau_{1}\right)}=\frac{\sqrt{b_{1}}}{\sqrt{b_{3}}-\sqrt{b_{1}}-\sqrt{b_{2}}}, \\
& \operatorname{Re}\left(\tau_{1}\right)= \pm \frac{\sqrt{\sqrt{b_{1}}\left(-b_{2} \sqrt{b_{3}}+\sqrt{b_{2}} b_{3}\right)-b_{1}\left(\sqrt{b_{2}} \sqrt{b_{3}}+\operatorname{Im}\left(\tau_{1}\right)^{2}\right)}}{\sqrt{b_{1}}},
\end{aligned}
$$

with $d_{0}=-\left(\sqrt{b_{1}}+\sqrt{b_{2}}-\sqrt{b_{3}}\right)^{2}$.

(ii) Solution 2

$$
\begin{aligned}
& \frac{\operatorname{Re}\left(\tau_{2}\right)}{\operatorname{Re}\left(\tau_{1}\right)}=-\frac{\operatorname{Im}\left(\tau_{2}\right)}{\operatorname{Im}\left(\tau_{1}\right)}=-\sqrt{\frac{b_{1}}{b_{2}},}, \quad \frac{\operatorname{Re}\left(\tau_{3}\right)}{\operatorname{Re}\left(\tau_{1}\right)}=-\frac{\operatorname{Im}\left(\tau_{3}\right)}{\operatorname{Im}\left(\tau_{1}\right)}=-\sqrt{\frac{b_{1}}{b_{3}}}, \\
& \frac{\operatorname{Re}(S)}{\operatorname{Re}\left(\tau_{1}\right)}=-\frac{\operatorname{Im}(S)}{\operatorname{Im}\left(\tau_{1}\right)}=\frac{\sqrt{b_{1}}}{\sqrt{b_{3}}-\sqrt{b_{1}}+\sqrt{b_{2}}}, \\
& \operatorname{Re}\left(\tau_{1}\right)= \pm \frac{\sqrt{-\sqrt{b_{1}}\left(b_{2} \sqrt{b_{3}}+\sqrt{b_{2}} b_{3}\right)+b_{1}\left(\sqrt{b_{2}} \sqrt{b_{3}}-\operatorname{Im}\left(\tau_{1}\right)^{2}\right)}}{\sqrt{b_{1}}}
\end{aligned}
$$

with $d_{0}=-\left(-\sqrt{b_{1}}+\sqrt{b_{2}}+\sqrt{b_{3}}\right)^{2}$.

(iii) Solution 3

$$
\begin{aligned}
& \frac{\operatorname{Re}\left(\tau_{2}\right)}{\operatorname{Re}\left(\tau_{1}\right)}=-\frac{\operatorname{Im}\left(\tau_{2}\right)}{\operatorname{Im}\left(\tau_{1}\right)}=-\sqrt{\frac{b_{1}}{b_{2}}}, \quad \frac{\operatorname{Re}\left(\tau_{3}\right)}{\operatorname{Re}\left(\tau_{1}\right)}=\frac{\operatorname{Im}\left(\tau_{3}\right)}{\operatorname{Im}\left(\tau_{1}\right)}=\sqrt{\frac{b_{1}}{b_{3}},} \\
& \frac{\operatorname{Re}(S)}{\operatorname{Re}\left(\tau_{1}\right)}=\frac{\operatorname{Im}(S)}{\operatorname{Im}\left(\tau_{1}\right)}=-\frac{\sqrt{b_{1}}}{\sqrt{b_{3}}+\sqrt{b_{1}}-\sqrt{b_{2}}}, \\
& \operatorname{Re}\left(\tau_{1}\right)= \pm \frac{\sqrt{\sqrt{b_{1}}\left(b_{2} \sqrt{b_{3}}-\sqrt{b_{2}} b_{3}\right)-b_{1}\left(\sqrt{b_{2}} \sqrt{b_{3}}+\operatorname{Im}\left(\tau_{1}\right)^{2}\right)}}{\sqrt{b_{1}}},
\end{aligned}
$$

with $d_{0}=-\left(\sqrt{b_{1}}-\sqrt{b_{2}}+\sqrt{b_{3}}\right)^{2}$.

(iv) Solution 4

$$
\begin{aligned}
& \frac{\operatorname{Re}\left(\tau_{2}\right)}{\operatorname{Re}\left(\tau_{1}\right)}=\frac{\operatorname{Im}\left(\tau_{2}\right)}{\operatorname{Im}\left(\tau_{1}\right)}=\sqrt{\frac{b_{1}}{b_{2}},}, \frac{\operatorname{Re}\left(\tau_{3}\right)}{\operatorname{Re}\left(\tau_{1}\right)}=\frac{\operatorname{Im}\left(\tau_{3}\right)}{\operatorname{Im}\left(\tau_{1}\right)}=\sqrt{\frac{b_{1}}{b_{3}},} \\
& \frac{\operatorname{Re}(S)}{\operatorname{Re}\left(\tau_{1}\right)}=-\frac{\operatorname{Im}(S)}{\operatorname{Im}\left(\tau_{1}\right)}=-\frac{\sqrt{b_{1}}}{\sqrt{b_{3}}+\sqrt{b_{1}}+\sqrt{b_{2}}}, \\
& \operatorname{Re}\left(\tau_{1}\right)= \pm \frac{\sqrt{\sqrt{b_{1}} \sqrt{b_{2}}\left(\sqrt{b_{1}}+\sqrt{b_{2}}+\sqrt{b_{3}}\right) \sqrt{b_{3}}-b_{1} \operatorname{Im}\left(\tau_{1}\right)^{2}}}{\sqrt{b_{1}}},
\end{aligned}
$$

with $d_{0}=-\left(\sqrt{b_{1}}+\sqrt{b_{2}}+\sqrt{b_{3}}\right)^{2}$.

(v) Solution 5

$$
\begin{aligned}
\operatorname{Im}\left(\tau_{1}\right) & =\sqrt{d_{0}}, \quad \operatorname{Re}\left(\tau_{1}\right)=0 \\
\operatorname{Im}\left(\tau_{2}\right) & =\sqrt{d_{0}-\operatorname{Re}\left(\tau_{2}\right)^{2}}, \quad \operatorname{Im}\left(\tau_{3}\right)=\sqrt{d_{0}-\operatorname{Re}\left(\tau_{3}\right)^{2}}, \\
\operatorname{Im}(S) & =\sqrt{d_{0}-\operatorname{Re}(S)^{2}}, \\
\operatorname{Re}(S) & =\frac{-d_{0}\left[d_{0} \operatorname{Im}\left(\tau_{3}\right)^{2}-\operatorname{Im}\left(\tau_{2}\right)^{2}\left(\operatorname{Im}\left(\tau_{3}\right)^{2}+\sqrt{\left(d_{0}-\operatorname{Im}\left(\tau_{2}\right)^{2}\right)\left(d_{0}-\operatorname{Im}\left(\tau_{3}\right)^{2}\right)}\right]\right.}{\sqrt{d_{0}-\operatorname{Im}\left(\tau_{2}\right)^{2}}\left(d_{0} \operatorname{Im}\left(\tau_{2}\right)^{2}+\left(d_{0}-\operatorname{Im}\left(\tau_{2}\right)^{2}\right) \operatorname{Im}\left(\tau_{3}\right)^{2}\right)},
\end{aligned}
$$

with $b_{1}=b_{2}=b_{3}=-d_{0}$. 
In these five solutions, $C P$-breaking and -conserving minima are degenerate. That is, the above five solutions always include the flat directions in the moduli space of axiodilaton and complex structure moduli, compared with the $C P$-conserving solution (31). We will study the origin of the above flat directions in the flux vacua from the viewpoint of modular symmetries in Sec. III E. The $C P$ breaking sources are then required to resolve the degeneracy between the $C P$-breaking and -conserving minima. Indeed, when we include the explicit $C P$-breaking term $b_{0}$ in the superpotential,

$$
\begin{aligned}
W= & a^{0} \tau_{1} \tau_{2} \tau_{3}+c^{1} S \tau_{2} \tau_{3}+c^{2} S \tau_{1} \tau_{3} \\
& +c^{3} S \tau_{1} \tau_{2}-\sum_{i=1}^{3} b_{i} \tau_{i}+d_{0} S-b_{0},
\end{aligned}
$$

it is possible to resolve the degeneracy between the $C P$ breaking and -conserving minima. Note that the moduli fields are denoted by $\tau_{i}, S$ rather than $\tau_{i}^{\prime}, S^{\prime}$. For instance, under the following flux set:

$$
\begin{gathered}
a^{0}=c^{1}=c^{2}=c^{3}=2, \quad b_{0}=d_{0}=-b_{3}=-2, \\
b_{1}=b_{2}=4
\end{gathered}
$$

leading to $n_{\text {flux }}=24$, all the complex structure moduli and axiodilaton are stabilized at a $C P$-breaking minimum,

$$
\begin{array}{ll}
\operatorname{Re}\left(\tau_{1}\right)=\operatorname{Re}\left(\tau_{2}\right) \simeq 0.127, & \operatorname{Re}\left(\tau_{3}\right)=\operatorname{Re}(S) \simeq 0.258 \\
\operatorname{Im}\left(\tau_{1}\right)=\operatorname{Im}\left(\tau_{2}\right) \simeq 0.958, & \operatorname{Im}\left(\tau_{3}\right)=\operatorname{Im}(S) \simeq 1.37
\end{array}
$$

with the masses squared of the moduli fields being positive,

$$
\mathcal{V}^{-2}(19,18,9.3,8.7,8.2,7.9,4.0,3.3)
$$

in the descending order.

\section{Even polynomials $(\gamma=\pi)$}

Next, we analyze the superpotential (20) consisting of polynomials of an even degree in the moduli fields. Similar to the analysis in Sec. III C 1, we redefine the moduli fields,

$$
\begin{array}{ll}
\tau_{1}=\frac{a^{1}}{c^{0} x_{s}} \tau_{1}^{\prime}, & \tau_{2}=\frac{a^{2}}{c^{0} x_{s}} \tau_{2}^{\prime}, \\
\tau_{3}=\frac{a^{3}}{c^{0} x_{s}} \tau_{3}^{\prime}, & S=x_{s} S^{\prime},
\end{array}
$$

simplifying the Kähler potential and the superpotential as

$$
\begin{aligned}
K & =-\ln (-i(S-\bar{S}))-2 \ln \mathcal{V}-\ln \left(i\left(\tau_{1}-\bar{\tau}_{1}\right)\left(\tau_{2}-\bar{\tau}_{2}\right)\left(\tau_{3}-\bar{\tau}_{3}\right)\right) \\
& =-\ln \left(-i\left(S^{\prime}-\bar{S}^{\prime}\right)\right)-2 \ln \mathcal{V}-\ln \left(i\left(\tau_{1}^{\prime}-\bar{\tau}_{1}^{\prime}\right)\left(\tau_{2}^{\prime}-\bar{\tau}_{2}^{\prime}\right)\left(\tau_{3}^{\prime}-\bar{\tau}_{3}^{\prime}\right)\right)-\ln \left(a^{1} a^{2} a^{3} /\left(c^{0}\right)^{3} x_{s}^{2}\right), \\
W & =-c^{0} S \tau_{1} \tau_{2} \tau_{3}-a^{1} \tau_{2} \tau_{3}-a^{2} \tau_{1} \tau_{3}-a^{3} \tau_{1} \tau_{2}+\sum_{i=1}^{3} d_{i} S \tau_{i}-b_{0} \\
& =-\left\{S^{\prime} \tau_{1}^{\prime} \tau_{2}^{\prime} \tau_{3}^{\prime}+\tau_{2}^{\prime} \tau_{3}^{\prime}+\tau_{1}^{\prime} \tau_{3}^{\prime}+\tau_{1}^{\prime} \tau_{2}^{\prime}\right\}+\sum_{i=1}^{3} d_{i}^{\prime} S^{\prime} \tau_{i}^{\prime}-b_{0}^{\prime},
\end{aligned}
$$

where we define

$$
x_{s}=\left(\frac{a^{1} a^{2} a^{3}}{\left(c^{0}\right)^{2}}\right)^{1 / 2}, \quad d_{i}^{\prime}=d_{i} \frac{a^{i}}{c^{0}}, \quad b_{0}^{\prime}=b_{0} .
$$

In analogy with the analysis in the previous section, we omit the prime symbols of fields unless specified otherwise. Then, we analyze the following Kähler potential and superpotential:

$$
\begin{aligned}
& K=-\ln (-i(S-\bar{S}))-2 \ln \mathcal{V}-\ln \left(i\left(\tau_{1}-\bar{\tau}_{1}\right)\left(\tau_{2}-\bar{\tau}_{2}\right)\left(\tau_{3}-\bar{\tau}_{3}\right)\right) \\
& W=-S \tau_{1} \tau_{2} \tau_{3}-\tau_{2} \tau_{3}-\tau_{1} \tau_{3}-\tau_{1} \tau_{2}+\sum_{i=1}^{3} d_{i} S \tau_{i}-b_{0},
\end{aligned}
$$

which is the simplified version of the superpotential with $c^{0}=a^{1}=a^{2}=a^{3}=1$.

Following the analysis in the previous subsection, we solve the redefined supersymmetric equations (30). It turns out that there exists a $C P$-conserving solution, 


$$
\begin{aligned}
& \operatorname{Re}\left(\tau_{1}\right)=\operatorname{Re}\left(\tau_{2}\right)=\operatorname{Re}\left(\tau_{3}\right)=\operatorname{Re}(S)=0, \quad \operatorname{Im}\left(\tau_{1}\right)=\left(-\frac{b_{0} d_{2} d_{3}}{d_{1}}\right)^{1 / 4}, \\
& \operatorname{Im}\left(\tau_{2}\right)=\left(-\frac{b_{0} d_{1} d_{3}}{d_{2}}\right)^{1 / 4}, \quad \operatorname{Im}\left(\tau_{3}\right)=\left(-\frac{b_{0} d_{1} d_{2}}{d_{3}}\right)^{1 / 4}, \quad \operatorname{Im}(S)=\left(-\frac{b_{0}}{d_{1} d_{2} d_{3}}\right)^{1 / 4},
\end{aligned}
$$

at which the masses of the moduli fields squared are positive. In addition, we find another solution:

(i) Solution 6

$$
\begin{aligned}
\operatorname{Im}\left(\tau_{1}\right) & =\sqrt{b_{0}-\operatorname{Re}\left(\tau_{1}\right)^{2}}, \\
\operatorname{Im}\left(\tau_{2}\right) & =\sqrt{b_{0}-\operatorname{Re}\left(\tau_{2}\right)^{2}}, \\
\operatorname{Im}\left(\tau_{3}\right) & =\sqrt{b_{0}-\operatorname{Re}\left(\tau_{3}\right)^{2}}, \\
\operatorname{Im}(S) & =\sqrt{\frac{1}{b_{0}}-\operatorname{Re}(S)^{2}}, \\
\operatorname{Re}\left(\tau_{1}\right) & =-b_{0} \frac{\operatorname{Re}\left(\tau_{2}\right)+\operatorname{Re}\left(\tau_{3}\right)+b_{0} \operatorname{Re}(S)+\operatorname{Re}\left(\tau_{2}\right) \operatorname{Re}\left(\tau_{3}\right) \operatorname{Re}(S)}{b_{0}+\operatorname{Re}\left(\tau_{2}\right) \operatorname{Re}\left(\tau_{3}\right)+b_{0} \operatorname{Re}(S)\left(\operatorname{Re}\left(\tau_{2}\right)+\operatorname{Re}\left(\tau_{3}\right)\right)},
\end{aligned}
$$

with $d_{1}=d_{2}=d_{3}=-b_{0}$.

As a result, there exist flat directions at the minimum due to the presence of unfixed moduli, and also $C P$-breaking and conserving minima are degenerate. In the same way as in Sec. III C 1, CP-breaking sources are required to lift the flat directions.

We perform the numerical search to find the supersymmetric $C P$-breaking minima in which the masses of all the moduli fields squared are positive, but we cannot find such a solution as indicated by our analytical expressions.

\section{Extension to other toroidal orbifolds}

So far, we have discussed the factorizable $T^{6} / \mathbb{Z}_{2}$ orientifold, which is also applicable to the $T^{6} /\left(\mathbb{Z}_{2} \times \mathbb{Z}_{2}{ }^{\prime}\right)$ orientifold, taking into account the proper tadpole cancellation conditions. In these $T^{6} / \mathbb{Z}_{2}$ and $T^{6} /\left(\mathbb{Z}_{2} \times \mathbb{Z}_{2}{ }^{\prime}\right)$ orientifolds, the largest number of flux quanta (three-cycles) is allowed under the orbifold projections. In this section, we discuss other toroidal orbifolds where the possible three-cycles (12) are restricted due to the orbifoldings.

There exists a single untwisted complex structure modulus on toroidal orbifolds, including the factorizable $T_{2} / \mathbb{Z}_{2}$, such as $T^{6} /\left(\mathbb{Z}_{2} \times \mathbb{Z}_{3}\right)=T^{6} / \mathbb{Z}_{6-I I}, T^{6} /\left(\mathbb{Z}_{2} \times \mathbb{Z}_{6}\right), T^{6} / \mathbb{Z}_{4}$, $T^{6} / \mathbb{Z}_{8-I I}$, and $T^{6} / \mathbb{Z}_{12-I I}[33-36]$, for the orbifolds preserving $\mathcal{N}=2$ supersymmetry, on which a part of the three-form basis in Eq. (12) remains under the orbifold projections [37]. ${ }^{4}$ On the other hand, in other orbifolds, (untwisted) complex structure moduli are fixed at discrete values. When we label the single untwisted complex

\footnotetext{
${ }^{4}$ Whether there exists a single complex structure modulus or not depends on lattices to construct $T^{6}$.
}

structure modulus $U$ on the above orbifolds, the 4D $C P$ invariance is preserved for the following superpotential via the procedure in Sec. III A:

(i) $\gamma=0(\bmod 2 \pi)$

$$
W=\xi_{1} U+\xi_{2} S
$$

(ii) $\gamma=\pi(\bmod 2 \pi)$

$$
W=\xi_{3} S U+\xi_{4},
$$

where $\xi_{1,2,3,4}$ are integers constrained by the quantization conditions of $F_{3}, H_{3}$. Given the Kähler potential of $U$,

$$
K=-\ln (-i(U-\bar{U})),
$$

the supersymmetric minimum is obtained by solving $D_{U} W=D_{S} W=0$,

(i) $\gamma=0(\bmod 2 \pi)$

$$
\operatorname{Re}(U)=-\frac{\xi_{2}}{\xi_{1}} \operatorname{Re}(S), \quad \operatorname{Im}(U)=\frac{\xi_{2}}{\xi_{1}} \operatorname{Im}(S),
$$

(ii) $\gamma=\pi(\bmod 2 \pi)$

$$
\begin{aligned}
& \operatorname{Re}(U)=-\frac{\xi_{4}}{\xi_{3}} \frac{\operatorname{Re}(S)}{\operatorname{Re}(S)^{2}+\operatorname{Im}(S)^{2}}, \\
& \operatorname{Im}(U)=-\frac{\xi_{4}}{\xi_{3}} \frac{\operatorname{Im}(S)}{\operatorname{Re}(S)^{2}+\operatorname{Im}(S)^{2}} .
\end{aligned}
$$

At the minima, there exists a flat direction in the moduli space of an axiodilaton and complex structure modulus, even for the restricted orbifolds. Hence, we conclude that 
Type IIB flux compactifications on toroidal orientifolds are not sufficient to realize the spontaneous $C P$ violation. Since flat directions generically appear at degenerate $C P$-breaking and -conserving minima, it is required to consider other $C P$-breaking sources which are discussed in Sec. III F.

\section{E. $C P$ and modular symmetry}

Before going to discuss the mechanism to lift the flat directions, we discuss the relationship among the 4D $C P$, the modular symmetry, and flat directions, which appear in the above potential analysis. In general, the presence of three-form fluxes breaks $S L(2, \mathbb{Z})_{S}$ and $S L(2, \mathbb{Z})_{i}$ modular symmetries associated with the axiodilaton $S$ and three complex structure moduli $\tau_{i}$ in the low-energy effective action, respectively, but it is possible to preserve subgroups of the modular groups for a specific choice of three-form fluxes. (For the classification of discrete modular symmetries, see Ref. [38].)

Let us focus on $T^{6} / \mathbb{Z}_{2}$ and $T^{6} /\left(\mathbb{Z}_{2} \times \mathbb{Z}_{2}^{\prime}\right)$ orientifolds with three complex structure moduli in Sec. III. After redefining the moduli fields as in Eqs. (29) and (44), threeform fluxes are expanded as

(i) $\gamma=0(\bmod 2 \pi)$

$$
\begin{aligned}
& \frac{1}{l_{s}^{2}} F_{3}=\alpha_{0}+b_{i}^{\prime} \beta^{i}=A_{i j}^{(1)} d \xi_{1}^{i} d \xi_{2}^{j} d x^{3}+A_{i j}^{(2)} d \xi_{1}^{i} d \xi_{2}^{j} d y^{3}, \\
& \frac{1}{l_{s}^{2}} H_{3}=\alpha_{i}+d_{0}^{\prime} \beta^{0}=A_{i j}^{(3)} d \xi_{1}^{i} d \xi_{2}^{j} d x^{3}+A_{i j}^{(4)} d \xi_{1}^{i} d \xi_{2}^{j} d y^{3},
\end{aligned}
$$

with $\xi_{i}=\left(y^{i}, x^{i}\right)^{T}$ and

$$
A_{i j}^{(1)}=\left(\begin{array}{cc}
-b_{3}^{\prime} & 0 \\
0 & 1
\end{array}\right), \quad A_{i j}^{(2)}=\left(\begin{array}{cc}
0 & -b_{2}^{\prime} \\
-b_{1}^{\prime} & 0
\end{array}\right), \quad A_{i j}^{(3)}=\left(\begin{array}{ll}
0 & 1 \\
1 & 0
\end{array}\right), \quad A_{i j}^{(4)}=\left(\begin{array}{cc}
d_{0}^{\prime} & 0 \\
0 & 1
\end{array}\right) .
$$

(ii) $\gamma=\pi(\bmod 2 \pi)$

$$
\begin{aligned}
& \frac{1}{l_{s}^{2}} F_{3}=a^{i} \alpha_{i}+b_{0} \beta^{0}=B_{i j}^{(1)} d \xi_{1}^{i} d \xi_{2}^{j} d x^{3}+B_{i j}^{(2)} d \xi_{1}^{i} d \xi_{2}^{j} d y^{3} \\
& \frac{1}{l_{s}^{2}} H_{3}=c^{0} \alpha_{0}+d_{i} \beta^{i},=B_{i j}^{(3)} d \xi_{1}^{i} d \xi_{2}^{j} d x^{3}+B_{i j}^{(4)} d \xi_{1}^{i} d \xi_{2}^{j} d y^{3}
\end{aligned}
$$

with

$$
B_{i j}^{(1)}=\left(\begin{array}{cc}
0 & 1 \\
1 & 0
\end{array}\right), \quad B_{i j}^{(2)}=\left(\begin{array}{cc}
b_{0}^{\prime} & 0 \\
0 & 1
\end{array}\right), \quad B_{i j}^{(3)}=\left(\begin{array}{cc}
-d_{3}^{\prime} & 0 \\
0 & 1
\end{array}\right), \quad B_{i j}^{(4)}=\left(\begin{array}{cc}
0 & -d_{2}^{\prime} \\
-d_{1}^{\prime} & 0
\end{array}\right) .
$$

When we consider the modular transformations on the tori $\left(T^{2}\right)_{i}$, the complex structure moduli as well as the coordinate of tori transform

$$
\tau_{i}^{\prime}=R_{i} \tau_{i}, \quad \xi_{i}^{\prime}=\left(R_{i}^{-1}\right)^{T} \xi_{i},
$$

with

$$
R_{i}=\left(\begin{array}{cc}
p_{i} & q_{i} \\
r_{i} & s_{i}
\end{array}\right) \in S L(2, \mathbb{Z})_{i}
$$

satisfying $p_{i} s_{i}-q_{i} r_{i}=1$. As discussed in detail in Ref. [38], the modular invariance of the effective action is realized when the three-forms $F_{3}$ and $H_{3}$ themselves are invariant under the modular transformations. Given the modular transformations on $\left(T^{2}\right)_{1} \times\left(T^{2}\right)_{2}$, the requirement for having the modular symmetries in the effective action leads to the modular transformations for the flux quanta in Eqs. (53) and (55),

$$
A^{(m)}=R_{1}^{-1} A^{(m)}\left(R_{2}^{-1}\right)^{T}, \quad B^{(m)}=R_{1}^{-1} B^{(m)}\left(R_{2}^{-1}\right)^{T},
$$

with $m=1,2,3,4$, where we use the transformations of $\xi_{i}$ in Eq. (56). Hence, the transformation matrices $R_{1,2}$ are constrained to satisfy the above relations for all $m$. From explicit expressions of $R_{2}$, 


$$
\begin{aligned}
R_{2} & =\left(A^{(m)}\right)^{T}\left(R_{1}^{-1}\right)^{T}\left(A^{(m)}\right)^{-1, T} \\
& =\left\{\left.\left(\begin{array}{cc}
s_{1} & b_{3}^{\prime} r_{1} \\
\frac{q_{1}}{b_{3}^{\prime}} & p_{1}
\end{array}\right)\right|_{m=1},\left.\left(\begin{array}{cc}
p_{1} & -\frac{b_{1}^{\prime} q_{1}}{b_{2}^{\prime}} \\
-\frac{b_{2}^{\prime} r_{1}}{b_{1}^{\prime}} & s_{1}
\end{array}\right)\right|_{m=2},\left.\left(\begin{array}{cc}
p_{1} & -q_{1} \\
-r_{1} & s_{1}
\end{array}\right)\right|_{m=3},\left.\left(\begin{array}{cc}
s_{1} & -d_{0}^{\prime} r_{1} \\
-\frac{q_{1}}{d_{0}^{\prime}} & p_{1}
\end{array}\right)\right|_{m=4}\right\}, \\
R_{2} & =\left(B^{(m)}\right)^{T}\left(R_{1}^{-1}\right)^{T}\left(B^{(m)}\right)^{-1, T} \\
& =\left\{\left.\left(\begin{array}{cc}
p_{1} & -q_{1} \\
-r_{1} & s_{1}
\end{array}\right)\right|_{m=1},\left.\left(\begin{array}{cc}
s_{1} & d_{3}^{\prime} r_{1} \\
\frac{q_{1}^{\prime}}{d_{3}^{\prime}} & p_{1}
\end{array}\right)\right|_{m=2},\left.\left(\begin{array}{cc}
s_{1} & -b_{0}^{\prime} r_{1} \\
-\frac{q_{1}^{\prime}}{b_{0}^{\prime}} & p_{1}
\end{array}\right)\right|_{m=3},\left.\left(\begin{array}{cc}
p_{1} & -\frac{d_{2}^{\prime} q_{1}}{d_{1}^{\prime}} \\
-\frac{d_{1}^{\prime} r_{1}}{d_{2}^{\prime}} & s_{1}
\end{array}\right)\right|_{m=4}\right\},
\end{aligned}
$$

we find that only the following flux quanta:

$$
\begin{aligned}
& d_{0}^{\prime}=-b_{3}^{\prime}=1, \quad b_{1}^{\prime}=b_{2}^{\prime} \quad(\gamma=0), \\
& d_{3}^{\prime}=-b_{0}^{\prime}=-1, \quad d_{1}^{\prime}=d_{2}^{\prime} \quad(\gamma=\pi)
\end{aligned}
$$

with $q_{1}=r_{1}, p_{1}=s_{1}$ or

$$
\begin{aligned}
& d_{0}^{\prime}=-b_{3}^{\prime}=-1, \quad b_{1}^{\prime}=b_{2}^{\prime} \quad(\gamma=0), \\
& d_{3}^{\prime}=-b_{0}^{\prime}=1, \quad d_{1}^{\prime}=d_{2}^{\prime} \quad(\gamma=\pi)
\end{aligned}
$$

with $q_{1}=-r_{1}, p_{1}=s_{1}$ are solutions of Eq. (58). Taking into account the condition $\operatorname{det}\left(R_{1}\right)=\operatorname{det}\left(R_{2}\right)=1$, the flux choice (60) corresponds to the trivial diagonal matrix for $R_{2}$ as well as $R_{1}$, whereas another choice (61) allows the $S$ transformation for the diagonal part of $S L(2, \mathbb{Z})_{1} \times S L(2, \mathbb{Z})_{2}$. We further analyze the modular transformation on $\left(T^{2}\right)_{3}$, which transforms the three-form fluxes $F_{3}$ and $H_{3}$ into

$$
\begin{aligned}
\left(\begin{array}{c}
F_{3} \\
H_{3}
\end{array}\right) & =\left(\begin{array}{ll}
\mathcal{C}_{i j}^{(2)} d \xi_{1}^{i} d \xi_{2}^{j} & \mathcal{C}_{i j}^{(1)} d \xi_{1}^{i} d \xi_{2}^{j} \\
\mathcal{C}_{i j}^{(4)} d \xi_{1}^{i} d \xi_{2}^{j} & \mathcal{C}_{i j}^{(3)} d \xi_{1}^{i} d \xi_{2}^{j}
\end{array}\right)\left(\begin{array}{c}
y^{3} \\
x^{3}
\end{array}\right) \\
& \rightarrow\left\{\left(\begin{array}{cc}
\mathcal{C}_{i j}^{(2)} d \xi_{1}^{i} d \xi_{2}^{j} & \mathcal{C}_{i j}^{(1)} d \xi_{1}^{i} d \xi_{2}^{j} \\
\mathcal{C}_{i j}^{(4)} d \xi_{1}^{i} d \xi_{2}^{j} & \mathcal{C}_{i j}^{(3)} d \xi_{1}^{i} d \xi_{2}^{j}
\end{array}\right)\left(R_{3}^{-1}\right)^{T}\right\}\left(\begin{array}{c}
y^{3} \\
x^{3}
\end{array}\right),
\end{aligned}
$$

with $\mathcal{C}_{i j}^{(m)}=\left\{A_{i j}^{(m)}, B_{i j}^{(m)}\right\}$, which is not invariant under $S L(2, \mathbb{Z})_{3}$ for a generic choice of fluxes. Indeed, $S$ and $T$ transformations of $\operatorname{SL}(2, \mathbb{Z})_{3}$ change the three-form fluxes $\left(F_{3}, H_{3}\right)$ themselves, namely,

$$
\left(\begin{array}{l}
F_{3} \\
H_{3}
\end{array}\right) \rightarrow\left\{\begin{array}{cc}
\left(\begin{array}{cc}
-\mathcal{C}_{i j}^{(1)} d \xi_{1}^{i} d \xi_{2}^{j} & \mathcal{C}_{i j}^{(2)} d \xi_{1}^{i} d \xi_{2}^{j} \\
-\mathcal{C}_{i j}^{(3)} d \xi_{1}^{i} d \xi_{2}^{j} & \mathcal{C}_{i j}^{(4)} d \xi_{1}^{i} d \xi_{2}^{j}
\end{array}\right)\left(\begin{array}{c}
y^{3} \\
x^{3}
\end{array}\right), & \text { with } R_{3}=\left(\begin{array}{cc}
0 & 1 \\
-1 & 0
\end{array}\right) \\
\left(\begin{array}{cc}
-\mathcal{C}_{i j}^{(1)} d \xi_{1}^{i} d \xi_{2}^{j}+\mathcal{C}_{i j}^{(2)} d \xi_{1}^{i} d \xi_{2}^{j} & \mathcal{C}_{i j}^{(1)} d \xi_{1}^{i} d \xi_{2}^{j} \\
-\mathcal{C}_{i j}^{(3)} d \xi_{1}^{i} d \xi_{2}^{j}+\mathcal{C}_{i j}^{(4)} d \xi_{1}^{i} d \xi_{2}^{j} & \mathcal{C}_{i j}^{(3)} d \xi_{1}^{i} d \xi_{2}^{j}
\end{array}\right)\left(\begin{array}{l}
y^{3} \\
x^{3}
\end{array}\right), \quad \text { with } R_{3}=\left(\begin{array}{ll}
1 & 1 \\
0 & 1
\end{array}\right)
\end{array}\right.
$$

which is not identical to the original $\left(F_{3}, H_{3}\right)$.

However, there is a chance to keep the (discrete) modular symmetry on $\left(T^{2}\right)_{3}$, identifying the modular transformation $R_{3}$ of $S L(2, \mathbb{Z})_{3}$ with $S L(2, \mathbb{Z})_{S}$ modular transformation $R$ associated with the axiodilaton. Note that $S L(2, \mathbb{Z})_{S}$ always exists in the effective action, where the axiodilaton as well as the three-form fluxes transform

$$
S \rightarrow R(S), \quad\left(\begin{array}{c}
F_{3} \\
H_{3}
\end{array}\right) \rightarrow R\left(\begin{array}{c}
F_{3} \\
H_{3}
\end{array}\right)
$$

for $R \in S L(2, \mathbb{Z})_{S}$. 
For the following choice of fluxes with

$$
\begin{array}{lll}
d_{0}^{\prime}=\mp b_{3}^{\prime}, & b_{1}^{\prime}=b_{2}^{\prime}= \pm 1, & (\gamma=0), \\
d_{3}^{\prime}=\mp b_{0}^{\prime}, & d_{1}^{\prime}=d_{2}^{\prime}= \pm 1, \quad(\gamma=\pi),
\end{array}
$$

the three-form fluxes $\left(F_{3}, H_{3}\right)$ allow us to identify $R$ with the element of $S L(2, \mathbb{Z})_{S}$ after performing the $S$ transformation of $S L(2, \mathbb{Z})_{3}$,

$$
\begin{aligned}
\left(\begin{array}{c}
F_{3} \\
H_{3}
\end{array}\right) & \rightarrow R R^{-1}\left(\begin{array}{cc}
-\mathcal{C}_{i j}^{(1)} d \xi_{1}^{i} d \xi_{2}^{j} & \mathcal{C}_{i j}^{(2)} d \xi_{1}^{i} d \xi_{2}^{j} \\
-\mathcal{C}_{i j}^{(3)} d \xi_{1}^{i} d \xi_{2}^{j} & \mathcal{C}_{i j}^{(4)} d \xi_{1}^{i} d \xi_{2}^{j}
\end{array}\right)\left(\begin{array}{c}
y^{3} \\
x^{3}
\end{array}\right) \\
& =R\left(\begin{array}{c}
F_{3} \\
H_{3}
\end{array}\right)
\end{aligned}
$$

with

$$
R=\left(\begin{array}{cc}
0 & \mp 1 \\
\pm 1 & 0
\end{array}\right)
$$

Here, the \pm signs are correlated in Eqs. (65) and (67). Hence, the $S$ transformation of a diagonal $S L(2, \mathbb{Z})_{3} \times$ $S L(2, \mathbb{Z})_{S}$ exists in the potential for a particular choice of fluxes, but it is difficult to realize the $T$ transformation of $S L(2, \mathbb{Z})_{3}$ even when the modular transformation of the axiodilaton is taken into account. The spontaneous symmetry breaking of these modular symmetries will be analyzed in the actual flux vacua later. Although we choose a particular basis of the three-form fluxes (52) and (54), namely $\left\{\xi_{1}, \xi_{2}\right\}$, it is possible to consider other bases, such as $\left\{\xi_{1}, \xi_{3}\right\}$ and $\left\{\xi_{2}, \xi_{3}\right\}$.

In the following, we analyze the modular symmetry of the flux vacua, which is the remnant of a modular symmetry in the effective action discussed so far. Remarkably, all the solutions we found in Sec. III satisfy the relation,

$$
\operatorname{Re}\left(\phi_{m}\right)^{2}+\operatorname{Im}\left(\phi_{m}\right)^{2}=1,
$$

with $\phi_{m}$ being the rescaled moduli for the solutions:

(i) Solution 1

$$
\phi_{1}=\left(\frac{b_{1}^{\prime}}{b_{2}^{\prime} b_{3}^{\prime}}\right)^{1 / 4} \frac{\tau_{1}^{\prime}}{p_{1}}, \quad \phi_{2}=\left(\frac{b_{2}^{\prime}}{b_{1}^{\prime} b_{3}^{\prime}}\right)^{1 / 4} \frac{\tau_{2}^{\prime}}{p_{1}}, \quad \phi_{3}=\left(\frac{b_{3}^{\prime}}{b_{1}^{\prime} b_{2}^{\prime}}\right)^{1 / 4} \frac{\tau_{3}^{\prime}}{p_{1}}, \quad \phi_{4}=\frac{p_{1} S^{\prime}}{\left(b_{1}^{\prime} b_{2}^{\prime} b_{3}^{\prime}\right)^{1 / 4}},
$$

with $p_{1} \equiv\left(-\sqrt{b_{1}^{\prime}}-\sqrt{b_{2}^{\prime}}+\sqrt{b_{3}^{\prime}}\right)^{1 / 2}$.

(ii) Solution 2

$$
\phi_{1}=\left(\frac{b_{1}^{\prime}}{b_{2}^{\prime} b_{3}^{\prime}}\right)^{1 / 4} \frac{\tau_{1}^{\prime}}{p_{2}}, \quad \phi_{2}=\left(\frac{b_{2}^{\prime}}{b_{1}^{\prime} b_{3}^{\prime}}\right)^{1 / 4} \frac{\tau_{2}^{\prime}}{p_{2}}, \quad \phi_{3}=\left(\frac{b_{3}^{\prime}}{b_{1}^{\prime} b_{2}^{\prime}}\right)^{1 / 4} \frac{\tau_{3}^{\prime}}{p_{2}}, \quad \phi_{4}=\frac{p_{2} S^{\prime}}{\left(b_{1}^{\prime} b_{2}^{\prime} b_{3}^{\prime}\right)^{1 / 4}},
$$

with $p_{2} \equiv\left(\sqrt{b_{1}^{\prime}}-\sqrt{b_{2}^{\prime}}-\sqrt{b_{3}^{\prime}}\right)^{1 / 2}$.

(iii) Solution 3

$$
\phi_{1}=\left(\frac{b_{1}^{\prime}}{b_{2}^{\prime} b_{3}^{\prime}}\right)^{1 / 4} \frac{\tau_{1}^{\prime}}{p_{3}}, \quad \phi_{2}=\left(\frac{b_{2}^{\prime}}{b_{1}^{\prime} b_{3}^{\prime}}\right)^{1 / 4} \frac{\tau_{2}^{\prime}}{p_{3}}, \quad \phi_{3}=\left(\frac{b_{3}^{\prime}}{b_{1}^{\prime} b_{2}^{\prime}}\right)^{1 / 4} \frac{\tau_{3}^{\prime}}{p_{3}}, \quad \phi_{4}=\frac{p_{3} S^{\prime}}{\left(b_{1}^{\prime} b_{2}^{\prime} b_{3}^{\prime}\right)^{1 / 4}},
$$

with $p_{3} \equiv\left(-\sqrt{b_{1}^{\prime}}+\sqrt{b_{2}^{\prime}}-\sqrt{b_{3}^{\prime}}\right)^{1 / 2}$.

(iv) Solution 4

$$
\phi_{1}=\left(\frac{b_{1}^{\prime}}{b_{2}^{\prime} b_{3}^{\prime}}\right)^{1 / 4} \frac{\tau_{1}^{\prime}}{p_{4}}, \quad \phi_{2}=\left(\frac{b_{2}^{\prime}}{b_{1}^{\prime} b_{3}^{\prime}}\right)^{1 / 4} \frac{\tau_{2}^{\prime}}{p_{4}}, \quad \phi_{3}=\left(\frac{b_{3}^{\prime}}{b_{1}^{\prime} b_{2}^{\prime}}\right)^{1 / 4} \frac{\tau_{3}^{\prime}}{p_{4}}, \quad \phi_{4}=\frac{p_{4} S^{\prime}}{\left(b_{1}^{\prime} b_{2}^{\prime} b_{3}^{\prime}\right)^{1 / 4}},
$$

with $p_{4} \equiv\left(\sqrt{b_{1}^{\prime}}+\sqrt{b_{2}^{\prime}}+\sqrt{b_{3}^{\prime}}\right)^{1 / 2}$.

(v) Solution 5

$$
\phi_{1}=\frac{\tau_{1}^{\prime}}{\sqrt{d_{0}^{\prime}}}, \quad \phi_{2}=\frac{\tau_{2}^{\prime}}{\sqrt{d_{0}^{\prime}}}, \quad \phi_{3}=\frac{\tau_{3}^{\prime}}{\sqrt{d_{0}^{\prime}}}, \quad \phi_{4}=\frac{S^{\prime}}{\sqrt{d_{0}^{\prime}}}
$$

(vi) Solution 6

$$
\phi_{1}=\frac{\tau_{1}^{\prime}}{\sqrt{b_{0}^{\prime}}}, \quad \phi_{2}=\frac{\tau_{2}^{\prime}}{\sqrt{b_{0}^{\prime}}}, \quad \phi_{3}=\frac{\tau_{3}^{\prime}}{\sqrt{b_{0}^{\prime}}}, \quad \phi_{4}=\sqrt{b_{0}^{\prime}} S^{\prime} .
$$


Here, we have explicitly written prime symbols in the solutions.

In this way, the flux vacua are distributed along the circumference of the unit circle as in Eq. (68) for the rescaled moduli. It means that when the rescaled moduli $\phi_{m}$ are equal to the original unrescaled moduli fields, some of $S$ transformations in $\Pi_{i=1}^{3} S L(2, \mathbb{Z})_{i} \times S L(2, \mathbb{Z})_{S}$ modular groups,

$$
\phi_{m} \rightarrow-\left(\phi_{m}\right)^{-1}=-\frac{\bar{\phi}_{m}}{\left|\phi_{m}\right|},
$$

with $\left|\phi_{m}\right|=1$ are manifest in the obtained flux vacua for a particular choice of fluxes. Furthermore, $S$ transformations in the flux vacua with $\left|\tau_{i}\right|=1$ correspond to the $C P$ transformations $\tau_{i} \rightarrow-\bar{\tau}_{i}$ as discussed in Sec. II. The presence of a $C P$ transformation at the flux vacua also supports the existence of unbroken 4D $C P$. Note that the solutions found in Sec. III C are parametrized by more generic fluxes, compared with the flux vacua having the (discrete) modular symmetry. To clarify this point, we show the solution 5 with the specific flux quanta $d_{0}^{\prime}=-b_{1}^{\prime}=-b_{2}^{\prime}=-b_{3}^{\prime}=1$ which lead to the following superpotential:

$$
W=\left\{\tau_{1}^{\prime} \tau_{2}^{\prime} \tau_{3}^{\prime}+S^{\prime} \tau_{2}^{\prime} \tau_{3}^{\prime}+S^{\prime} \tau_{1}^{\prime} \tau_{3}^{\prime}+S^{\prime} \tau_{1}^{\prime} \tau_{2}^{\prime}\right\}+\sum_{i=1}^{3} \tau_{i}^{\prime}+S^{\prime}
$$

The explicit form of the solution 5 is given by

$$
\operatorname{Re}\left(\phi_{m}\right)^{2}+\operatorname{Im}\left(\phi_{m}\right)^{2}=d_{0}^{\prime}=1,
$$

where $\phi_{m}=\left\{\tau_{i}^{\prime}, S^{\prime}\right\}$ are distributed along the circumference of the unit circle. However, the above solutions do not satisfy the condition (65), meaning that only the $S$ transformation of the diagonal $S L(2, \mathbb{Z})_{1} \times S L(2, \mathbb{Z})_{2}$ remains in the flux vacua. Thus, the flat directions are remnant of the modular symmetries in rescaled moduli. Furthermore, the 4D $C P$ can be embedded into a modular symmetry in the background of three-form fluxes such that the flat directions correspond to the unit circle in the unrescaled moduli. The other choices of three-form fluxes give rise to the circle with different radii, and the $4 \mathrm{D} C P$ is not identified with the $S$ transformation.

\section{F. Comments on the $\boldsymbol{C P}$-breaking sources}

In this section, we comment on sources of $C P$ violation to resolve the degeneracy between $C P$-breaking and -conserving minima.

So far, we have focused on the complex structure moduli and dilaton, but the unfixed Kähler moduli whose axions determine the size of the $\theta$ term still play an important role of $C P$ violation and affect the dynamics of the other moduli. To stabilize the Kähler moduli, we introduce the nonperturbative effects to the superpotential,

$$
W=\gamma(\tau, S) e^{-f T},
$$

where $T$ denotes the Kähler moduli appearing in the gauge kinetic function (2). Here, $\gamma(\tau, S)$ is supposed to the oneloop determinant of the D3-brane instanton effects or the one-loop corrections to the gauge couplings on the $D 7$-branes, where the gauginos condensate. Although the lifting of flat directions in the $C P$-breaking and -conserving minima is highly dependent on the functional form of $\gamma(\tau, S)$ as well as the mechanism to uplift the anti-de Sitter vacuum obtained after having fixed all the moduli to the de Sitter one, it might lead to the realization of spontaneous $C P$ violation even when $\gamma(\tau, S)$ is a $C P$-invariant function.

Another possibility to realize the spontaneous $C P$ violation is to consider Calabi-Yau threefolds or blowing up the orbifolds as an extension of toroidal orbifolds. It is interesting to discuss the Calabi-Yau compactifications with three-form fluxes, but the number of complex structure moduli is typically of $\mathcal{O}(100)$, which is hard to analyze the $C P$-breaking minima analytically. Also, the introduction of geometric fluxes" as well as nongeometric fluxes will be useful to realize the spontaneous $C P$ violation because such fluxes induce couplings between the Kähler moduli and complex structure moduli [39-41]. We leave the detailed study of these possibilities for future work.

\section{CONCLUSIONS}

In this paper, we systematically investigated whether the spontaneous $C P$ violation can be realized in Type IIB flux compactifications on toroidal orientifolds, which allow for the stabilization of the moduli in a controlled way.

Similar to the heterotic string theory, 4D CP is regarded as a discrete gauge symmetry embedded into the 10D proper Lorentz symmetry. To ensure the presence of 4D CP in the potential, the three-cycles three-form fluxes turn on are restricted to even or odd polynomials with respect to the complex structure moduli associated with tori and the axiodilaton. Our detailed analysis for a generic choice of fluxes shows that there exist flat directions, where $C P$ breaking and -conserving vacua are degenerate. Hence, the flux compactifications are insufficient to realize the spontaneous $C P$ violation in Type IIB toroidal orientifolds. The statement also holds for the heterotic string theory as well as Type IIA string theory on toroidal orbifolds with fluxes because the functional form of the flux-induced superpotential is restricted to one of the Type IIB flux compactifications. These flat directions are remnants of the modular $S$ transformation for rescaled moduli. Furthermore, the 4D $C P$ is embedded into the modular symmetries in the effective action for a particular choice of fluxes, and such an approach to unify $C P$ and modular 
symmetries is closely related to the recent discussion in the heterotic orbifold context [7-10] but in a different context.

To resolve the degeneracy between the $C P$-breaking and -conserving vacua, an extension of our system is required to introduce nonperturbative effects with respect to the Kähler moduli and/or (non)geometric fluxes in the superpotential. Calabi-Yau compactifications as well as the blown up orbifold compactifications will give a crucial role of lifting the flat directions. We will report this interesting work in the future.

Recently, modular flavor symmetries were studied to derive realistic quark and lepton mass matrices. (See, e.g., Ref. [42].) Although the modular symmetry is completely broken at a generic value of $\tau$, its subgroups remain at fixed points, e.g., $Z_{3}$ symmetry at $\tau=e^{i \pi / 3}, Z_{2}$ symmetry at $\tau=i$, and $Z_{2}$ symmetry at $\tau=i \infty$. In this sense, moduli values at and near these fixed points are interesting. (See, e.g., Ref. [43].) For example, realistic quark and lepton mass matrices are obtained around $\tau=i$ [44]. Our analysis can lead to such values, i.e., $\tau_{1}=i$ for $d_{0}=1$ in solution 5 and $\tau_{1,2}=0.127+0.958 i$ in Eq. (39) as well as $\tau \sim i \infty$ for sufficiently large $d_{0}$ in solution 5 . Our result would also be important from this viewpoint.

\section{ACKNOWLEDGMENTS}

T. K. was supported in part by MEXT KAKENHI Grant No. JP19H04605. H. O. was supported in part by JSPS KAKENHI Grants No. JP19J00664 and No. JP20K14477.
[1] C. Abel et al. (nEDM Collaboration), Phys. Rev. Lett. 124, 081803 (2020).

[2] M. Dine, R. G. Leigh, and D. A. MacIntire, Phys. Rev. Lett. 69, 2030 (1992).

[3] K. Choi, D. B. Kaplan, and A. E. Nelson, Nucl. Phys. B391, 515 (1993).

[4] A. Strominger and E. Witten, Commun. Math. Phys. 101, 341 (1985).

[5] J. Giedt, Mod. Phys. Lett. A 17, 1465 (2002).

[6] T. Kobayashi and C. S. Lim, Phys. Lett. B 343, 122 (1995).

[7] H. P. Nilles, M. Ratz, A. Trautner, and P. K. S. Vaudrevange, Phys. Lett. B 786, 283 (2018).

[8] T. Dent, Phys. Rev. D 64, 056005 (2001).

[9] A. Baur, H. P. Nilles, A. Trautner, and P. K. S. Vaudrevange, Phys. Lett. B 795, 7 (2019).

[10] A. Baur, H. P. Nilles, A. Trautner, and P. K. S. Vaudrevange, Nucl. Phys. B947, 114737 (2019).

[11] T. Kobayashi, Y. Shimizu, K. Takagi, M. Tanimoto, T. H. Tatsuishi, and H. Uchida, Phys. Rev. D 101, 055046 (2020).

[12] T. Kobayashi and H. Otsuka, Phys. Lett. B 807, 135554 (2020).

[13] F. Feruglio, C. Hagedorn, and R. Ziegler, J. High Energy Phys. 07 (2013) 027.

[14] M. Holthausen, M. Lindner, and M. A. Schmidt, J. High Energy Phys. 04 (2013) 122.

[15] M. C. Chen, M. Fallbacher, K. T. Mahanthappa, M. Ratz, and A. Trautner, Nucl. Phys. B883, 267 (2014).

[16] P. Novichkov, J. Penedo, S. Petcov, and A. Titov, J. High Energy Phys. 07 (2019) 165.

[17] D. Lust, P. Mayr, R. Richter, and S. Stieberger, Nucl. Phys. B696, 205 (2004).

[18] D. Lust, S. Reffert, and S. Stieberger, Nucl. Phys. B706, 3 (2005).

[19] D. Lust, S. Reffert, and S. Stieberger, Nucl. Phys. B727, 264 (2005).

[20] D. Cremades, L. Ibanez, and F. Marchesano, J. High Energy Phys. 07 (2003) 038.
[21] M. Cvetic and I. Papadimitriou, Phys. Rev. D 68, 046001 (2003); 70, 029903(E) (2004).

[22] S. A. Abel and A. W. Owen, Nucl. Phys. B663, 197 (2003).

[23] D. Cremades, L. Ibanez, and F. Marchesano, J. High Energy Phys. 05 (2004) 079.

[24] T. Kobayashi, K. Nishiwaki, and Y. Tatsuta, J. High Energy Phys. 04 (2017) 080.

[25] T. Kobayashi, S. Nagamoto, S. Takada, S. Tamba, and T. H. Tatsuishi, Phys. Rev. D 97, 116002 (2018).

[26] T. Kobayashi and S. Tamba, Phys. Rev. D 99, 046001 (2019).

[27] S. Kachru, M. B. Schulz, and S. Trivedi, J. High Energy Phys. 10 (2003) 007.

[28] S. Gukov, C. Vafa, and E. Witten, Nucl. Phys. B584, 69 (2000); B608, 477(E) (2001).

[29] A. Hanany and B. Kol, J. High Energy Phys. 06 (2000) 013.

[30] E. Witten, J. High Energy Phys. 02 (1998) 006.

[31] R. Blumenhagen, B. Kors, D. Lust, and S. Stieberger, Phys. Rep. 445, 1 (2007).

[32] Y. Honma and H. Otsuka, J. High Energy Phys. 04 (2020) 001.

[33] L. E. Ibanez, J. Mas, H. P. Nilles, and F. Quevedo, Nucl. Phys. B301, 157 (1988).

[34] A. Font, L. E. Ibanez, and F. Quevedo, Phys. Lett. B 217, 272 (1989).

[35] Y. Katsuki, Y. Kawamura, T. Kobayashi, N. Ohtsubo, Y. Ono, and K. Tanioka, Nucl. Phys. B341, 611 (1990).

[36] T. Kobayashi and N. Ohtsubo, Int. J. Mod. Phys. A 09, 87 (1994).

[37] D. Lust, S. Reffert, W. Schulgin, and S. Stieberger, Nucl. Phys. B766, 68 (2007).

[38] T. Kobayashi and H. Otsuka, Phys. Rev. D 101, 106017 (2020).

[39] S. Gurrieri, J. Louis, A. Micu, and D. Waldram, Nucl. Phys. B654, 61 (2003).

[40] S. Kachru, M. B. Schulz, P. K. Tripathy, and S. P. Trivedi, J. High Energy Phys. 03 (2003) 061. 
[41] J. Shelton, W. Taylor, and B. Wecht, J. High Energy Phys. 10 (2005) 085.

[42] F. Feruglio, arXiv:1706.08749; T. Kobayashi, K. Tanaka, and T. H. Tatsuishi, Phys. Rev. D 98, 016004 (2018); J. T. Penedo and S. T. Petcov, Nucl. Phys. B939, 292 (2019); J. C. Criado and F. Feruglio, SciPost Phys. 5, 042 (2018); T. Kobayashi, N. Omoto, Y. Shimizu, K. Takagi, M. Tanimoto, and T. H. Tatsuishi, J. High Energy Phys. 11 (2018) 196; P. P. Novichkov, J. T. Penedo, S. T. Petcov, and A. V. Titov, J. High Energy Phys. 04 (2019) 005; 04 (2019) 174; H. Okada and M. Tanimoto, Phys. Lett. B 791, 54
(2019); T. Kobayashi, Y. Shimizu, K. Takagi, M. Tanimoto, T. H. Tatsuishi, and H. Uchida, Phys. Lett. B 794, 114 (2019).

[43] F. J. de Anda, S. F. King, and E. Perdomo, Phys. Rev. D 101, 015028 (2020); P. P. Novichkov, S. T. Petcov, and M. Tanimoto, Phys. Lett. B 793, 247 (2019); I. de Medeiros Varzielas, S. F. King, and Y. Zhou, Phys. Rev. D 101, 055033 (2020); S. F. King and Y. Zhou, Phys. Rev. D 101, 015001 (2020); G. Ding, S. F. King, X. Liu, and J. Lu, J. High Energy Phys. 12 (2019) 030.

[44] H. Okada and M. Tanimoto, arXiv:1905.13421. 